\title{
DETERMINACIÓN DEL CONSUMO MÁXIMO DE OXÍGENO DEL FUTBOLISTA COSTARRICENSE DE PRIMERA DIVISIÓN EN PRETEMPORADA 2008
}

\author{
Braulio Sánchez y Jorge Salas \\ Universidad Nacional \\ Programa Ciencias del Ejercicio y la Salud \\ brau09@hotmail.com,josaca8@yahoo.com
}

\begin{abstract}
RESUMEN
El objetivo de este estudio fue valorar el perfil cardiorrespiratorio ( $\mathrm{VO}_{2}$ máx.) de los jugadores de fútbol de la primera división de Costa Rica en pretemporada y comparar el VO2 máx., según puestos. Metodología: en este estudio se evaluó 9 equipos de la primera división del fútbol en Costa Rica, para una muestra de 219 jugadores profesionales entre los 20 y los 36 con una edad promedio de 24.64 años \pm 4.35 de edad; un promedio de peso corporal $73.34 \mathrm{Kg} \pm$ 7.34 y un porcentaje de grasa de $9.78 \% \pm 3.64$. Para evaluar el VO2 máx. de los jugadores, se usó un protocolo de rampa en banda sin fin según recomendaciones planteadas por Wilmore y Costill (2007), la prueba fue de carácter máxima. Resultados: se encontró un $\mathrm{VO}_{2}$ máx., promedio de $57.71 \mathrm{ml} / \mathrm{kg} / \mathrm{min} \pm 8.8$. Por otro lado, no se encontró diferencias estadísticamente significativas $(p=.752)$ entre puestos. Conclusión: no hubo diferencias en el $\mathrm{VO}_{2}$ máx., por puestos.
\end{abstract}

PALABRAS CLAVES: fútbol, capacidad cardiorrespiratoria, rendimiento deportivo.

\section{INTRODUCCIÓN}

El rendimiento superior de los deportistas, es el resultado de una compleja combinación de diversos factores y es muy posible que el factor más importante a la hora de determinar el potencial de un deportista sea la dotación genética. Asimismo, otro aspecto que tiene un profundo efecto sobre el rendimiento es la cantidad e idoneidad del entrenamiento previo a las competiciones (MacDougall, Wenger y Green, 1995).

Alba (2005), cita que los científicos deportivos no pueden cambiar los factores hereditarios, pero por medio de pruebas de laboratorio y campo pueden controlar los progresos y la prescripción del rendimiento. De acuerdo con MagDougall et al. (1995), el $\mathrm{VO}_{2}$ máx., es el principal indicador de las posibilidades aeróbicas del examinado, debido a que integra múltiples funciones orgánicas (ventilatorios, cardiovasculares, sanguíneos, musculares), por lo cual tiene una estrecha relación con el nivel de acondicionamiento y con el estado de salud.

Teniendo en cuenta que el $\mathrm{VO}_{2}$ máx., se define según Wilmore y Costill (2007) y MacDogall et al. (1995), como el ritmo más alto de consumo de oxígeno alcanzado, durante la realización de ejercicios máximos o agotadores, entonces este límite, el $\left(\mathrm{VO}_{2}\right.$ máx.) dicta la intensidad del esfuerzo o el ritmo que se puede sostener en el ejercicio, estos autores aclaran que se puede seguir realizando ejercicio durante un corto tiempo después de alcanzar el $\mathrm{VO}_{2}$ máx., movilizando las reservas anaeróbicas, pero estas tienen también una capacidad finita. 
URL www.una.ac.cr/mhsalud

Ha quedado demostrado según MacDogall et al. (1995), y Wilmore y Costill (2007) que, con el entrenamiento un adulto sano joven y relativamente desentrenado puede mejorar el $\mathrm{VO}_{2}$ máx. entre un $15 \%$ y el $20 \%$ e incluso más, dependiendo de sus niveles previos de entrenamiento.

Wilmore y Costill (2007), indican que estas mejoras permiten ejecutar actividades de resistencia con un nivel de esfuerzo más alto o a un ritmo más rápido, mejorando el potencial de rendimiento.

Según Alba (2005), es importante citar que el $\mathrm{VO}_{2}$ máx., es ideal en etapa competitiva del ciclo de entrenamiento de futbolistas. Asimismo, este autor, menciona que en futbolistas el $\mathrm{VO}_{2}$ máx., debería ser de $58 \mathrm{ml} / \mathrm{Kg}$./min, lo cual se encuentra en el rango establecido por de MacDogall et al. (1995), quienes lo sitúan entre $50-70 \mathrm{ml} / \mathrm{kg} / \mathrm{min}$.

Según MacDogall (1995), las evaluaciones de los diferentes componentes y características asociadas con la potencia aeróbica pueden ser útiles a la hora de determinar los siguientes puntos: 1) La preparación actual de un deportista o su idoneidad para realizar una actividad determinada. 2) El énfasis que se debe poner en el entrenamiento aeróbico y en otros tipos de entrenamiento. 3) El tipo de entrenamiento aeróbico que debe prevalecer. 4) El tiempo que debe durar el énfasis 5) La magnitud y el ritmo de cambio generados por un programa de entrenamiento determinado. 6) El ritmo y el patrón o estrategia a utilizar en la competición. También, es importante tomar en cuenta que la detección de problemas médicos, nutricionales, de crecimiento o de exceso de entrenamiento, pues puede estar limitando el rendimiento en la competición en particular y su rendimiento aeróbico en general.

Para Vargas (2007), la obtención del $\mathrm{VO}_{2}$ máx., se puede realizar de forma indirecta o forma directa, la primera a través de un test indirecto, el cual tiene debe ser válido, objetivo y fiable, ejemplo test de los 12 minutos de carrera, la segunda mediante una medición de gases realizada con un analizador telemétrico.

Este mismo autor (2007), indica que en el fútbol el $\mathrm{VO}_{2}$ máx., no siempre determina el rendimiento deportivo de un futbolista, ya que existen otros factores que afectan este rendimiento como: los contenidos técnicos, tácticos, psicológicos y sociales de la acción futbolística.

No obstante, autores como Garrido y González (2006) y Arecheta, Gómez y Lucía (2006); indican que el $\mathrm{VO}_{2}$ máx., es de suma importancia para un mayor desempeño físico y una mejor planificación del entrenamiento, tanto en temporada como en pretemporada. Bajo estos lineamientos autores como Silvestre, West, Maresh y Kraemer (2006) han reportado el valor $\mathrm{VO}_{2}$ máx., de acuerdo al puesto en jugadores categoría sub 20, ya que la planificación debe cumplir con esta especificidad, los porteros presentaron un $\mathrm{VO}_{2}$ máx., $55.94 \pm 5.78 \mathrm{ml} / \mathrm{kg} / \mathrm{min}$., los defensas $57.20 \pm 9.86 \mathrm{ml} / \mathrm{kg} / \mathrm{min}$., los mediocampistas $58.38 \pm 9.85$ y los delanteros $57.87 \pm 5.94$ $\mathrm{ml} / \mathrm{kg} / \mathrm{min}$., en términos generales reportan un $\mathrm{VO}_{2}$ máx., de $57.71 \pm 8.80 \mathrm{ml} / \mathrm{kg} / \mathrm{min}$.

Es evidente que en el entrenamiento deportivo moderno, no solo aspectos físicos son importantes, pero las evidencias revisadas demuestran la importancia que en el nivel de la preparación física tiene la determinación del $\mathrm{VO}_{2}$ máx., para orientar el trabajo de los futbolistas. Sin embargo, pese a esta importancia los estudios realizados con futbolistas no son tan frecuentes y menos en el medio latinoamericano. Por ende, el propósito del presente estudio con base en los hallazgos anteriormente citados, fue determinar el consumo de oxígeno en futbolistas costarricenses en pretemporada y comparar el $\mathrm{VO}_{2}$ máx., según puesto. 
URL www.una.ac.cr/mhsalud

\section{METODOLOGÍA:}

Sujetos: participaron en el estudio 219 jugadores de la primera división del fútbol de Costa Rica, de los cuales 23 eran porteros, 57 defensas, 94 volantes y 45 delanteros. La edad promedio de los participantes fue de $24.64 \pm 4.35$ años. La participación se realizó por invitación, se invitaron a los 12 equipos de primera división, de los cuales aceptaron participar en las evaluaciones 9 equipos, cuyos nombres se reservan para garantizar el anonimato de las instituciones deportivas. Los jugadores recibieron información previa a su participación en las pruebas, las cuales constituyeron parte de las valoraciones planeadas por los cuerpos médicos de cada equipo (para lo cual esta investigación sirvió como un apoyo para estas instituciones deportivas, mediante la entrega a estas de reportes de los resultados de las evaluaciones). Además contó con el permiso por parte de los clubes para utilizar los datos con fines de redactar artículos científicos.

Instrumentos y materiales: Para el este estudio se utilizó un analizador de gases marca Medgraphics modelo VO2000 (con un .98 de validez y 95\% de confiabilidad) y una banda sin fin marca Hill-Med.

Procedimiento: cada uno de los sujetos fue evaluado bajo el siguiente protocolo recomendado por MacDougall et al. (1995) en banda sin fin y fue de carácter máximo. En cada etapa se aumenta la velocidad en 1 milla $(1.609 \mathrm{~km})$, cada etapa tarda 2 minuto, ninguna presenta porcentaje de inclinación en la banda.

\begin{tabular}{ccc}
\hline Etapa & Tiempo & Velocidad $(\mathrm{km} / \mathrm{h})$ \\
\hline Calentamiento & $3 \mathrm{~min}$ & 7.0 \\
1 & $2 \mathrm{~min}$ & 8.2 \\
2 & $2 \mathrm{~min}$ & 9.4 \\
3 & $2 \mathrm{~min}$ & 10.6 \\
\hline
\end{tabular}

Nota: ejemplo de como se incrementa la velocidad por etapa, el número de etapas no tiene límite.

Todos los sujetos fueron sometidos al protocolo al máximo esfuerzo, hasta alcanzar el $\mathrm{VO}_{2}$ máx., el cual se determinó mediante los cambios en los valores del $\mathrm{VO}_{2} / \mathrm{VCO} 2$ mostrados por el RQ, el cual debió alcanzar el valor igual o mayor a 1.

Análisis estadístico: se aplicó estadística descriptiva (promedios y desviaciones estándar), y además como estadística inferencial se aplicó un ANOVA de una vía para muestras independientes, con un Post Hoc de Scheffe, para comparar el $\mathrm{VO}_{2}$ máx., según el puesto en que juega cada futbolista. Los análisis se corrieron con el paquete estadístico SPSS versión 8.0 para Windows. 
URL www.una.ac.cr/mhsalud

\section{RESULTADOS:}

Tabla 1. Promedios y desviaciones estándar (DE) por puesto para la variable consumo máximo de oxígeno

\begin{tabular}{lcc}
\hline \multicolumn{1}{c}{ Puesto } & $\begin{array}{c}\text { Promedio } \\
(\mathrm{ml} / \mathrm{Kg} / \mathrm{min})\end{array}$ & $\mathrm{n}$ \\
\hline Porteros & $55.94 \pm 5.78$ & 23 \\
Defensas & $57.20 \pm 9.86$ & 57 \\
Volantes & $58.38 \pm 9.85$ & 94 \\
Delanteros & $57.87 \pm 5.94$ & 45 \\
Total & $\mathbf{5 7 . 7 1} \pm \mathbf{8 . 8 0}$ & 219 \\
\hline
\end{tabular}

En la tabla anterior se resaltan los resultados de la muestra en total $(\mathrm{n}=219)$ presentando un promedio general de $57.71 \pm 8.8 \mathrm{ml} / \mathrm{kg} / \mathrm{min}$, siendo los volantes quienes presentan un $\mathrm{VO}_{2}$ máx., más alto, y los porteros con el valor del $\mathrm{VO}_{2}$ máx., más bajo, sin embargo no se observó diferencias estadísticamente significativas entres los distintos puestos $(\mathrm{p}=.752, \mathrm{~F}=.477)$.

\section{DISCUSIÓN}

Como se pudo apreciar el promedio de $\mathrm{VO}_{2}$ máx., de los jugadores costarricenses en pretemporada, concuerdo con lo esperado para futbolistas de alto nivel según lo planteado por Alba (2005), Moreira (2008) y MacDogall et al. (1995) en términos generales, en el caso de cada puesto presentan un comportamiento muy similar con lo reportado por Silvestre et al. (2006).

Por otra parte Wilmore y Costill (2007), indican que un adecuado nivel de consumo de oxígeno, permite ejecutar actividades de resistencia con un nivel de esfuerzo más alto o a un ritmo más rápido, mejorando el potencial de rendimiento. Sin embargo, Reilly (1996) menciona que otro aspecto importante es el límite superior al cual se puede sostener un ejercicio continuo. Este límite está influenciado por el denominado umbral anaeróbico y por la alta utilización fraccional del $\mathrm{VO}_{2}$ máx. Este mismo autor indica que en el fútbol se utiliza un consumo de oxígeno correspondiente al $75 \%$ del $\mathrm{VO}_{2}$ máx. Valor probablemente cercano al umbral anaeróbico en los futbolistas de alto nivel, esto sugiere hacer más investigaciones para determinar la tolerancia del futbolista costarricense a trabajos cerca o por encima de su umbral anaeróbico.

Además, de lo encontrado en este estudio, es necesario la determinación del consumo máximo de oxígeno no solo en pretemporada sino también y como lo establece la literatura al final de cada periodo competitivo MacDougall et al. (1995).

Si se analizan los resultados obtenidos y haciendo referencia la tabla 1 , no se encontraron diferencias estadísticamente significativas entre puestos, esto se puede explicar porque al inicio de la pretemporada, la capacidad aeróbica no ha sido estimulada suficientemente, dando como resultado una homogeneidad de los jugadores en este parámetro. No obstante, conociendo la naturaleza de cada puesto, estos resultados variarán con el proceso de entrenamiento conforme se alcance la especificidad requerida para cada puesto, tal y como lo ilustra Reilly (1996), quien indica que en promedio un portero recorre durante el juego $4 \mathrm{~km}$, los defensas de $8.5 \mathrm{~m}$, volantes de $10.2 \mathrm{~km}$ y delanteros de $8.8 \mathrm{~km}$, quedando claro que la capacidad aeróbica debe variar en función del puesto, durante el periodo competitivo. 
URL www.una.ac.cr/mhsalud

En síntesis, los resultados muestran que los futbolistas costarricenses de primera división evaluados, presentan al inicio de la pretemporada un $\mathrm{VO}_{2}$ máx., normal, según lo encontrado en otros estudios con futbolistas internacionales. Sin embargo, es recomendable extender este tipo de análisis más allá de la pretemporada, para observar si se logra mejorar específicamente, este indicador, en el periodo competitivo, según las demandas especificas de cada puesto.

Agradecimientos: colaboradores de la investigación: Henry Sánchez Villalobos, Juan Manuel Ramírez Madrigal, Cristian Azofeifa Mora, José Andrés Trejos Montoya, José Sánchez Salazar, Steven Jara Oviedo, Carlos Camacho, Jorge Valerio, Geancarlo Gamboa Salas.

\section{BIBLIOGRAFÍA}

Alba, A. (2005). Test funcionales: cineantropometría y prescripción del entrenamiento en el deporte y la actividad fisica. (2 ed.). Colombia: Editorial Kinesis.

Arecheta, C., Gómez, M. y Lucía, A. (2006). La importancia del $\mathrm{VO}_{2}$ máx. para realizar esfuerzos intermitentes de alta intensidad en el fútbol femenino de élite. Revista Kronos, 5 (9), 4 12 .

Garrido, R.P. y González, M. (2006). Volumen de oxígeno por kilogramo de masa muscular en futbolistas. Revista Internacional de Medicina y Ciencias de la Actividad Física y del Deporte, 6(21), 44-61.

MacDougall, D. J., Wenger, H. A. y Green, H. (1995). Evaluación fisiológica del deportista. (2 ed). Barcelona, España: Editorial Paidotribo.

Moreira, S. B. (2008). The Evolution of the Individual Factors Performance in the Elite of the World Soccer, between 1970 and 1998. Fitness and Performance Journal, 7 (2), 107-111

Reilly, T. (1996). Aspectos fisiológicos del fútbol. Actualización en Ciencias del Deporte, 4 (13). Recuperado de http://www.sobreentrenamiento.com/Publice/Articulo.asp?ida=165

Silvestre, R., West, C., Maresh, C. y Kraemer, W. (2006). Body Composition and Physical Performance in Men's Soccer: a Study of a National Collegiate Athletic Association Division I Team. Journal of Strength and Conditioning Research, 20(1), 177-183.

Vargas, C. (2007). Consumo de oxígeno máximo telemétrico vs. Yo-Yo Endurance Test, en jugadores del fútbol profesional argentino. Revista de entrenamiento deportivo, 21 (4), 13-18.

Wilmore, J. y Costill, D. (2007). Fisiología del esfuerzo y del deporte (6 ed.). Barcelona, España: Editorial Paidotribo.

Fecha de recepción del artículo: 8 de octubre del 2008.

Fecha de aceptación del artículo: 22 de setiembre del 2009.

Fecha de publicación del artículo: 31 de diciembre del 2009. 